\title{
Silencing PD-1 and PD-L1 with nanoparticle-delivered small interfering RNA increases cytotoxicity of tumor-infiltrating lymphocytes
}

\author{
Yanheng $\mathrm{Wu}^{1}$, Wenyi Gu**,1, Jiang $\mathrm{Li}^{2}$, Chen $\mathrm{Chen}^{3}$ \& Zhi Ping Xü,1 \\ ${ }^{1}$ Australian Institute for Bioengineering \& Nanotechnology, University of Queensland, St Lucia, QLD 4072, Australia \\ ${ }^{2}$ State Key Laboratory of Oncology in South China, Sun Yat-Sen University, Guangzhou, China \\ ${ }^{3}$ School of Biomedical Sciences, Queensland Brain Institute, University of Queensland, St Lucia, QLD 4072, Australia \\ *Author for correspondence: Tel.: +61 7334 63809; gordonxu@uq.edu.au \\ **Author for correspondence: Tel.: +61 7 33463814; Fax: +61 7 33463973; w.gu@uq.edu.au
}

Aim: To determine if silencing PD-1 on tumor-infiltrating lymphocytes (TILs) and its ligand-1 (PD-L1) on cancer cells will enhance the cytotoxicity of TILs. Materials \& methods: Lipid-coated calcium phosphate nanoparticles were synthesized to deliver siRNAs against PD-1 and PD-L1 to TILs and breast cancer MCF7 cells. The downregulation of PD-1/PD-L1 expressions was determined by real-time PCR and western blotting assays. The killing efficacy of TILs to MCF-7 cells was determined by cytotoxic T lymphocyte assay. Results: Lipid-coated calcium phosphate nanoparticles effectively delivered siRNAs and silenced PD-1 and $P D$-L1sh expression. The knockdown of either gene or both greatly improved the cytotoxicity of TILs. Conclusion: Silencing PD-1 and PD-L1 is an effective approach to increase TIL cytotoxicity to cancer cells.

First draft submitted: 10 July 2018; Accepted for publication: 4 January 2019; Published online: 22 March 2019

Keywords: breast cancer • cytokines • MCF-7 • nanoparticles delivery • PD-1 • PD-L1 • siRNA • TILs

\section{Background}

Over the past decades, the rapid advancement in cancer therapy provides life-saving options for cancer patients. Cancer immunotherapy is one of the most promising approaches in current cancer treatment. As one significant part of immunotherapy, adoptive cell transfer is making great breakthroughs in cancer treatment, which was initially reported in the 1980s [1]. Adoptive cell transfer relies on immune cell populations that mediate direct tumoricidal effects, including conventional cytotoxic $\mathrm{CD}^{+} \mathrm{T}$ lymphocytes (CTLs), given alone or together with helper $\mathrm{CD} 4^{+}$ T cells, natural killer (NK) cells, 'cytokine-induced killer' cells and chimeric antigen receptor T-cell [2,3]. Chimeric antigen receptor $\mathrm{T}$ cell therapy has been successful in treating patients with hematologic malignancies, but it has been less effective in treating solid tumors [4]. For NK and cytokine-induced killer cells and CTLs-based therapies, though they can work in some solid tumors, they are not tumor antigen specific sensitized and the killing efficiency for cancer cells is not high. On the other hand, tumor-infiltrating lymphocyte (TIL)-mediated therapy becomes more popular as these cells are tumor antigen specific and have higher killing ability for solid tumors like renal cell carcinoma, breast cancer, oral squamous cell carcinoma and non-small-cell lung cancer [5-8].

TIL-based therapy is also a personalized treatment that can be achieved based on expanding these lymphocytes $e x$ vivo from surgically excised tumor specimens of patients and then adoptively transferring back into the patient [9]. However, tumor-specific TILs can be inactivated in vivo by cellular factors that suppress their activation and effector functions, such as T-regulatory and myeloid-derived suppressor cells, or by signaling pathways through immune checkpoint molecules such as PD-1 on TIL interaction with its ligand PD-L1 (also known as B7-H1) expressed on tumor cells. There is a growing body of work suggesting that TILs are restrained in vivo by immunosuppressive molecules, such as TIM-3, LAG3, PD-1 and CTLA-4 [5,7,8]. In particular, expression of the negative regulator, PD-L1 on tumor cells inhibits the activation of $\mathrm{T}$ cells upon binding to its receptor PD-1, thereby preventing effectiveness of antitumor immunity [10-13]. The expression of PD-1 and PD-L1 is associated with poor prognosis 
and cancer recurrence in breast cancer patients [14,15]. Moreover, the high-level expression of PD-1 is usually seen in late stage cancer patients [14].

Blockade of these negative regulators on $\mathrm{T}$ cells in the tumor microenvironment may improve antitumor $\mathrm{T}$ cell responses and lead to improved immunotherapeutic outcomes for cancer patients. There are several antibodies and inhibitors developed in recent years, including nivolumab (Opdivo, Bristol-Myers Squibb), pembrolizumab (Keytruda, Merck \& Co. Inc.) and atezolizumab (Tecentriq, Roche Genetech). Although inhibitors and antibodies against cell surface molecules like anti-CD40L, CTLA4-Ig, PD-1 and PD-L1 can inhibit these negative regulators, they have a short half-life and need multiple treatments [16]. Accordingly, the repeating use leads to high costs as well as high rates of adverse effects [17].

RNAi-based therapeutics have been developed as a potential novel class of therapeutic agent to treat many human diseases including cancer [18-20]. Small RNA molecules such as siRNA and miRNA are highly effective for cancer therapies due to their ability to specifically silence the expression of cancer-related genes or selectively regulate the pathways that are involved in the development and progression of malignancy. However, RNAi therapy for the treatment of cancer has issues of stability and delivery [21]. Thus, a safe and effective delivery system especially for $\mathrm{T}$ cells is required for effective RNAi therapy.

The lipid-coated calcium phosphate nanoparticles (LCP NPs) are a kind of nonviral vectors, which have been widely applied in delivery of siRNA [22], chemical drug [23] and cDNA plasmid [24]. This is because LCP NPs have a versatile formulation depending on the application aims. For this nanomaterial, calcium phosphate (CaP) cores are prepared first and coated with a single 1,2-dioleoyl-sn-glycero-3-phosphate (sodium salt; DOPA) layer. After this, the CaP-DOPA cores are further coated with an additional lipid layer as an outer leaflet to form an asymmetric lipid bilayer [24]. For successful endosome escape, the cationic 1,2-dioleoyl-3-trimethylammonium-propane (DOTAP) lipid is often added as an outer leaflet to destabilize the endosomes [24,25]. LCP NPs have been studied and proved to be a powerful tool to deliver siRNA [22,26-28].

In this study, we investigated whether knocking down of $P D-1$ in TILs or $P D-L 1$ in cancer cells and simultaneously knocking down both could improve antitumor immune responses of TILs in an ex vivo model. The objectives of this research were: to effectively deliver PD-1 and PD-L1 siRNAs to TILs and cancer cells and silence the target genes using LCP NPs; to confirm the enhanced killing efficiency of TILs to breast cancer cells after downregulation of either PD-1 or PD-L1and simultaneous both on TILs and breast cancer cell (MCF-7); and to reveal the mechanism of enhancing the killing efficiency through understanding the regulation of the relevant cytokines. Our data confirm that the downregulation of PD-1 or PD-L1 or both can significantly enhance T-cell immunity (cytotoxicity) against breast cancer cells and has an important potential implication for TIL-based immunotherapy for cancer patients in the future.

\section{Materials \& methods \\ Chemicals}

Sample preparation was performed under sterile conditions. Double-stranded DNA labeled with cyanine 3 (Cy3-dsDNA) was purchased from GeneWorks (SA, Australia). Phospholipids (DOPA, 1,2-dioleoyl-sn-glycero-3phosphocholine (DOPC) and DOTAP) were purchased from Avanti Polar Lipid (AL, USA). The siRNAs for PD-1 and PD-L1 knockdown and other chemicals and reagents were purchased from Sigma-Aldrich (NSW, Australia) if not illustrated specifically. Water used in experiments was de-ionized Milli-Q water.

\section{LCP NP preparation}

LCP NPs were prepared by a modified two-step method based on the previous reports [26,28]. The anionic lipid-coated $\mathrm{CaP}$ core was synthesized by a water-in-oil microemulsion method, and then the CaP core was coated with a second lipid layer to form the bilayer LCP NPs by the film-rehydration method. Briefly, the first microemulsion (solution 1a) was prepared by mixing $75 \mu \mathrm{l}$ of $5 \mathrm{M} \mathrm{CaCl}_{2}$ and $50 \mu \mathrm{l}$ of $\mathrm{H}_{2} \mathrm{O}$ with $5 \mathrm{ml}$ of premixed cyclohexane/Igepal CO-520 (70/30, v/v). The second microemulsion (solution 1b) containing sodium phosphate was prepared by adding $75 \mu \mathrm{l}$ of $50 \mathrm{mM} \mathrm{Na}_{2} \mathrm{HPO}_{4}$ and $50 \mu \mathrm{l}$ of $\mathrm{H}_{2} \mathrm{O}$ into another $5 \mathrm{ml}$ of oil phase. Then, the second microemulsion was added with $50 \mu \mathrm{l}$ of $20 \mathrm{mM}$ DOPA in chloroform and then with the first microemulsion drop by drop, followed by stirring for another $15 \mathrm{~min}$. The CaP-DOPA cores were harvested by adding $10 \mathrm{ml}$ of absolute ethanol and centrifuging at $10,000 \mathrm{~g}$ for $20 \mathrm{~min}$ and washed with $10 \mathrm{ml}$ of ethanol three-times. The collected $\mathrm{CaP}$ core particles were then dispersed in $1 \mathrm{ml}$ of $\mathrm{CHCl}_{3}$ and mixed with DOTAP/DOPC/cholesterol (2:1:3) in $\mathrm{CHCL}_{3}$. After evaporation under reduced pressure, the lipid film was then hydrated in phosphate buffered 
saline (PBS) buffer ( $\mathrm{pH}$ 7.4) to obtain LCP NPs, which were normally well dispersed under gentle ultrasound treatment.

Loading siRNA/dsDNA into LCP NPs

Cy3-dsDNA or siRNA was encapsulated in LCP NPs by premixing an equal amount of Cy3-dsDNA or siRNA $(15 \mu \mathrm{l} 100 \mu \mathrm{M})$ in both $\mathrm{CaCl}_{2}$ and $\mathrm{Na}_{2} \mathrm{HPO}_{4}$ solutions $(75 \mu \mathrm{l})$. The following procedure for making LCP-Cy3dsDNA NPs or LCP-siRNA NPs was the same as described in above section.

The efficiency of dsDNA encapsulation and the dsDNA loading capacity in LCP NPs was determined as follows. After LCP-Cy3-dsDNA particles were dissolved in the lysis buffer (2 mM EDTA and 0.05\% Triton X-100 in pH 7.8 Tris buffer) by incubating at $65^{\circ} \mathrm{C}$ for $10 \mathrm{~min}$, the concentration of Cy3-dsDNA released from dissolved LCP NPs was determined by measuring the fluorescence intensity, and then the amount of dsDNA loaded into the LCP NPs was calculated. All data are reported as the mean value \pm standard deviation in three parallel experiments.

\section{Characterization of LCP NPs}

To visualize the morphology of LCP NPs, the NP suspension was dropped on to a 300 mesh carbon-coated copper grid and dried on a filter paper at room temperature. The grid was then observed in a transmission electron microscope (TEM, JEM-3010, ZEOL, Tokyo, Japan). The hydrodynamic diameter and $\zeta$-potential of LCP NPs were determined by using a dynamic light scattering device at room temperature (DLS, Zetasizer Nano, Malvern, UK).

\section{TILs isolation \& characterization}

Fresh tumor samples were obtained from 15 breast cancer patients. All the patients were provided written consent forms before being subjected to blood and/or tumor collection. All the patient-related work was approved by the Research Ethics Committee of the Sun-Yat-Sen University Cancer Center (Guangzhou, China).

TILs were isolated from breast cancer biopsy specimens by mincing the tissue into small pieces and digesting them with collagenase type IV $(0.1 \mathrm{mg} / \mathrm{ml})$ (Sigma-Aldrich) for $2 \mathrm{~h}$, followed by culture in X-VIVO-15 medium (Lonza, Sydney, Australia) containing 5\% human AB serum and recombinant human IL-2 (150 IU/ml) in 24-well plates, followed by an expansion using a rapid expansion protocol (REP) [29,30]. Once the sufficient number of T cells $\left(>1 \times 10^{7}\right)$ was generated, they were cryopreserved for later expansion. A REP for 'young TILs' that was previously used in melanoma patients was followed. Cryopreserved, pre-REP TILs from patients were thawed and further expanded to treatment levels using an anti-CD3 antibody (OKT-3, $30 \mathrm{ng} / \mathrm{ml}$, R\&D Systems, MN, USA), rhIL-2 (BD PharMingen, Hong Kong, China) and irradiated feeder cells, as previously described [30].

\section{Fluorescence-activated cell sorting (FACS) analysis}

Fluorescence-activated cell sorting (FACS) analysis was performed with a BD Accuri ${ }^{\mathrm{TM}} \mathrm{C} 6$ (CA, USA) flow cytometer and CFlow Sampler software (Becton Dickinson, CA, USA). The immunophenotypes and the PD-1 expression level of TILs were determined using FACS as reported previously [6]. The PD-L1 expression levels in MCF-7 cells were similarly analyzed by FACS as described before [31]. Briefly, to make sure the positive cell populations are representative, during analysis the dead cells were first excluded from all samples and the single cells were gated on FSC-A (area) against FSC-W (width). Single cells were then plotted for positive populations against negative and isotype controls.

\section{Knockdown of PD-1 in TILs \& PD-L1 in MCF-7 with siRNAs delivered by LCP NPs}

The siRNAs used in the study were synthesized by Sigma-Aldrich. The sequences were: $P D-1$ siRNA: sense $5^{\prime}-$ AGAccuuGAuAcuuucAAAd-TsdT-3' and antisense 5'-UUUGAAAGuAUcAAGGUCUdTsdT-3'; PD-L1 siRNA: sense 5'-AGAcG-uAAGcAGuGuuGAAdTsdT-3' and antisense 5'-UUcAAcACUGCUuACGUCUdTsdT-3'.

Each siRNA was encapsulated in LCP NPs for cell treatment, and was named LCP-si-PD-1 and LCP-si-PD-L1 respectively. For TILs, $1 \times 10^{6}$ cells were seeded in a well of 6 -well plate and mixed with LCP-Si-PD- 1 at $37^{\circ} \mathrm{C}$ for $4 \mathrm{~h}$. The culture medium was replaced with the fresh medium and transfected TILs were cultured for another $72 \mathrm{~h}$ at $37^{\circ} \mathrm{C}, 5 \% \mathrm{CO}_{2}$. For MCF-7 cells, $1 \times 10^{5}$ cells were seeded in a well of 6-well plate and mixed with LCP-Si-PD-L1 at $37^{\circ} \mathrm{C}$ for $4 \mathrm{~h}$. Similarly, the culture medium was replaced with the fresh medium and transfected MCF-7 cells were cultured for another $72 \mathrm{~h}$ at $37^{\circ} \mathrm{C}, 5 \% \mathrm{CO}_{2}$. After transfection, PD-1 and PD-L1 expression levels were determined by real-time (RT) PCR, and FACS, respectively. 
Enzyme-linked immunosorbent assay (ELISA)

Cytokines such as IFN- $\gamma$, TNF- $\alpha$, IL-17 and IL-10 secretion from T cells were determined utilizing the relevant ELISA kits (R\&D Systems) according to the supplier's protocols.

Real-time PCR

All RT-PCR assays were conducted according to the manufacturer's instructions. In brief, $1.2 \mathrm{ml}$ of trizol/chloroform $(1: 5, \mathrm{v} / \mathrm{v})$ was added to lyse cells and the supernatant was collected by centrifugation (12,500 r.p.m., $15 \mathrm{~min})$. Then, $2.4 \mathrm{ml}$ of isopropanol was added and the supernatant was centrifuged for another $15 \mathrm{~min}$ at 12,500 r.p.m. The collected pellet was washed with $70 \%$ ethanol. After drying the pellet, $50 \mu \mathrm{l}$ of $\mathrm{H}_{2} \mathrm{O}$ was added to resuspend the total RNA. Reverse transcription reactions were performed in $20 \mu \mathrm{l}$ as the manufacturer instructed and the cDNA was diluted with $80 \mu \mathrm{l}$ of $\mathrm{H}_{2} \mathrm{O}$. For each well, $3.5 \mu \mathrm{l}$ of cDNA solution mixed with $8.5 \mu \mathrm{l}$ of PCR Master-Mixture. After centrifugation, RT-PCR was conducted (Real Time PCR, iCycler iQ ${ }^{T M}$, Bio-Rad, CA, USA).

\section{TILs killing efficacy determination}

The killing efficacy of TILs to MCF-7 was determined by CTL assay. MCF-7 cells (PD-L1+) treated with LCPSi-PD-L1 (40 nM) was named MCF-7 ${ }^{\mathrm{K}}$ (PD-L1-). TILs (PD-1+) treated with LCP-Si-PD-1 (80 nM) was named TILs $^{\mathrm{K}}$ (PD-1-). To prepare target cancer cells, MCF-7 and MCF-7 ${ }^{\mathrm{K}}$ cells were seeded at a density of $1 \times 10^{4}$ cells/well in U-bottomed 96-well microplates, cultured for $18 \mathrm{~h}$, and the medium was then replaced by $50 \mu \mathrm{l}$ fresh phenol red-free Roswell Park Memorial Institute (RPMI) 1640 medium (Gibco-BRL) containing 5\% fetal calf serum (FCS). To prepare the pretreated effector cells, TILs and TILs ${ }^{\mathrm{k}}$ were incubated for $24 \mathrm{~h}$ in CNE2$\mathrm{CM}$ and then collected and resuspended in phenol red-free RPMI 1640 containing 5\% FCS. Fifty microliters of the suspensions were added to each well at various $\mathrm{T}$ cell/cancer cell ratios of 10:1, 30:1, 100:1. After $4 \mathrm{~h}$ incubation, the plates were centrifuged and $50 \mu \mathrm{l}$ of the supernatant was transferred to new 96-well flat-bottomed plates, cytotoxic activity of TILs was evaluated by standard lactate dehydrogenase release assay using CytoTox 96 ${ }^{\circledR}$ Non-Radioactive Cytotoxicity Assay (Promega, WI, USA) in U-bottomed 96-well microplates, following the manufacturer's instructions.

\section{Statistical analysis}

Data presented as the mean \pm standard error of mean (SEM) or the mean \pm standard error (SE) were analyzed by one-way ANOVA with Tukey post-hoc test to compare all groups, or Student's t-test was used to compare two groups using GraphPad Prism software. A p-value $<0.05$ was considered significant. ${ }^{*} \mathrm{p}<0.05$; ${ }^{* *} \mathrm{p}<0.01$.

\section{Results}

\section{LCP NPs \& dsDNA loading}

As shown in Figure 1A, the prepared LCP NPs were sphere-like particles with the typical hollow structure in CaP cores, as previously reported $[26,28]$. The average hydrodynamic particle diameter of LCP NPs was approximately $30 \mathrm{~nm}$ after coated with the second lipid layer (Figure 1B), with a $\zeta$-potential of $+18 \mathrm{mV}$. The positive charge is mainly attributed to the positively charged lipid DOTAP co-coated on the external layers, as described previously [32].

When loaded with Cy3-dsDNA into LCP NPs, the average hydrodynamic particle size was kept unchanged, with a $\zeta$-potential of $+14 \mathrm{mV}$. The slight reduction of the $\zeta$-potential is mainly due to the excess $\mathrm{dsDNA}$ in the core, whose negative charge is not all balanced by the matrix cations, as discussed previously [26,28]. Quantitative analysis indicated that dsDNA loading efficiency was about $50 \%$, comparable with previous reports [33], and the dsDNA/siRNA loading capacity was approximately $58 \mu \mathrm{g} / \mathrm{mg}$ (LCP). The loading capacity of siRNA was then calculated based on the dsDNA loading.

\section{Determination of PD-1/PD-L1 expression on isolated TILs \& MCF-7}

The TILs were isolated from breast cancer patients and then expanded (Table 1). The PD-1 or PD-L1 expression levels on expanded TILs or MCF-7 cells were measured by flow cytometry analysis. A typical analysis of patient BC011 samples was shown in Figure 2A and C. There were about $41 \% \mathrm{CD}^{+}$and $36 \% \mathrm{CD}^{+} \mathrm{T}$ cells in the expanded TIL population. These T cells were found to have a high level of PD-1 expression. As shown in Figure $2 \mathrm{~B}$ and $\mathrm{D}, 71 \% \mathrm{CD}^{+}$and $68 \% \mathrm{CD}^{+} \mathrm{T}$ cells were PD-1 positive. As also shown in Table 1 , all expanded TILs collected from 15 breast cancer patients of stage 3 or above (pT3 or pT4), the samples contained $\mathrm{CD}^{+}$and $\mathrm{CD} 8^{+}$ 


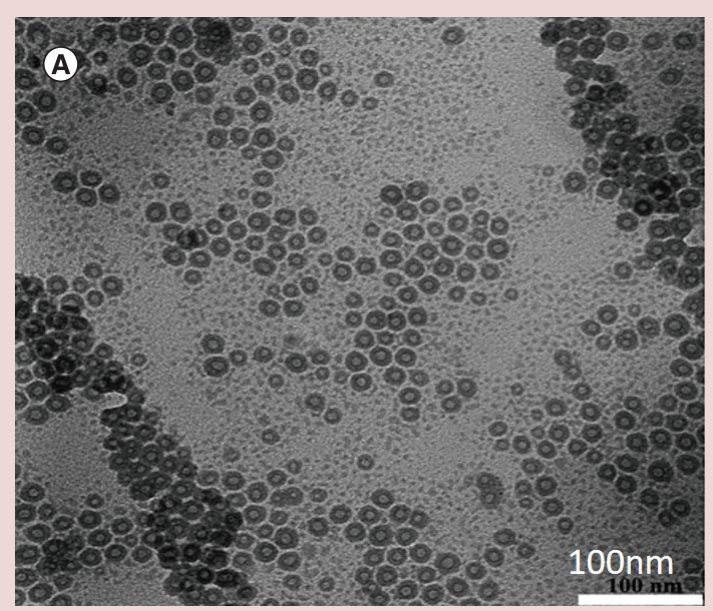

(B)

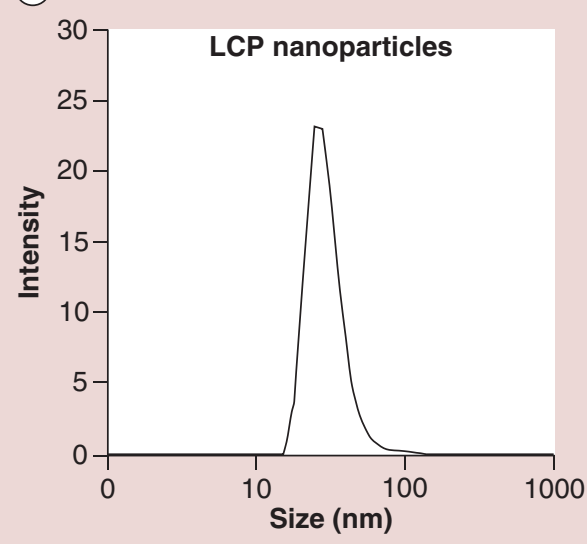

Figure 1. Characterization of lipid-coated calcium phosphate nanoparticles. (A) Transmission electron microscope image of LCP nanoparticles, the scale bar represents $100 \mathrm{~nm}$; (B) the size distribution of LCP nanoparticles.

LCP: Lipid-coated calcium phosphate.

\begin{tabular}{|c|c|c|c|c|c|}
\hline Patient & Age & Tumor stage & Lymph node involvement & Tumor grade & $\begin{array}{l}\text { CD8 }{ }^{+} \text {PD- } 1^{+} \text {positive cell } \\
\text { percentage }(\%)\end{array}$ \\
\hline BC001 & 54 & рт3 & $\mathrm{pN} 1$ & 2 & 23 \\
\hline BC002 & 57 & pT2 & pNO & 2 & 31 \\
\hline BC003 & 76 & pT4 & $\mathrm{pN} 2$ & 3 & 38 \\
\hline BC004 & 44 & pT2 & $\mathrm{pN} 1$ & 2 & 17 \\
\hline BC007 & 48 & pT1 & pNO & 1 & 19 \\
\hline BC008 & 42 & pT2 & $\mathrm{pN} 1$ & 2 & 31 \\
\hline BC009 & 63 & pT2 & $\mathrm{pN} 1$ & 2 & 29 \\
\hline BC010 & 39 & pT2 & pNO & 1 & 22 \\
\hline BC015 & 52 & рT3 & pN2 & 3 & 35 \\
\hline
\end{tabular}

$\dagger^{\mathrm{BC}} 011$ is the representative breast cancer case mainly involved in this study.

T cells with a relatively higher level of PD-1 expression. Thus, the current research on the TILs collected from BC011 patient would be used as a representative for this group of patients.

For this study, we used breast cancer MCF-7 cells as the cancer cell model. These cells expressed reasonable PD-L1 level, with $31 \%$ being PD-L1 positive (Figure 2E). This level of PD-L1 expression in MCF-7 would also represent the specific situation for breast cancers, where $15-20 \%$ cancer cells are PD-L1 positive [15]. Therefore, TILs and MCF-7 cells are both good representatives to test our hypothesis.

\section{LCP-si-PD-1 silencing PD-1 in TILs}

The expanded BC011 TILs were then treated with LCP-siRNA-PD-1 to knockdown the expression of PD-1 for both mRNA and protein levels. As shown in Figure 3A, compared with the control, PD-1 mRNA percentage was significantly decreased from 86 to $18 \%$ when the siRNA concentration increased from 20 to $160 \mathrm{nM}$. In Figure 3B, the PD-1 protein level was also remarkably reduced at $40 \mathrm{nM}$ siRNA and was nearly totally inhibited at $160 \mathrm{nM}$ 


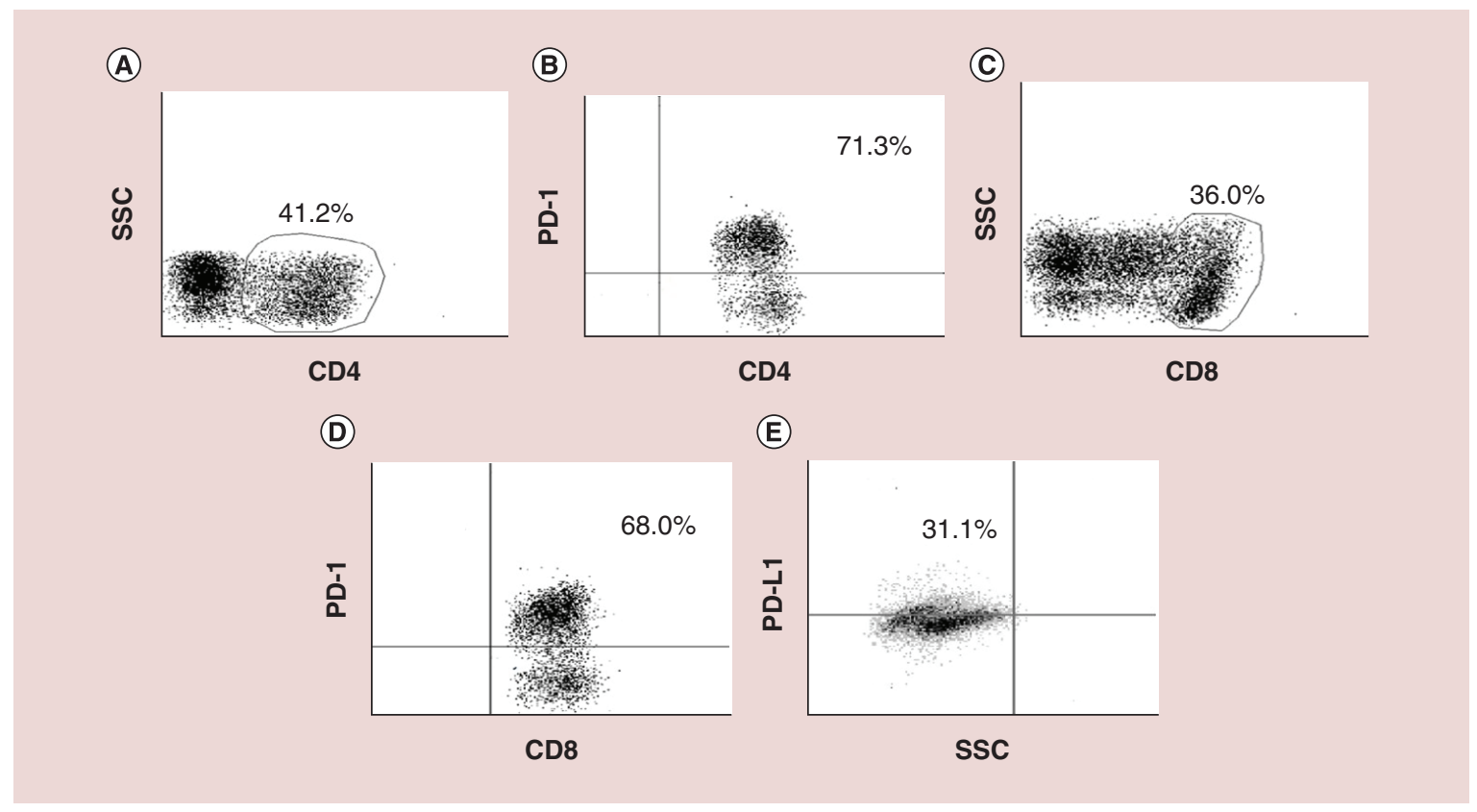

Figure 2. Representative flow cytometry data for PD-1 expression on tumor-infiltrating lymphocytes and PD-L1 expression on MCF-7 cells. (A) Sorting of CD4 ${ }^{+} \mathrm{T}$ cells and (B) PD-1 $1^{+}$cells in $\mathrm{CD} 4^{+} \mathrm{T}$ cells. (C) Sorting of $\mathrm{CD} 8^{+} \mathrm{T}$ cells and (D) PD- $1^{+}$cells in CD8 ${ }^{+}$T cells. (E) PD-L1+ cells in MCF-7 cells.

(A)

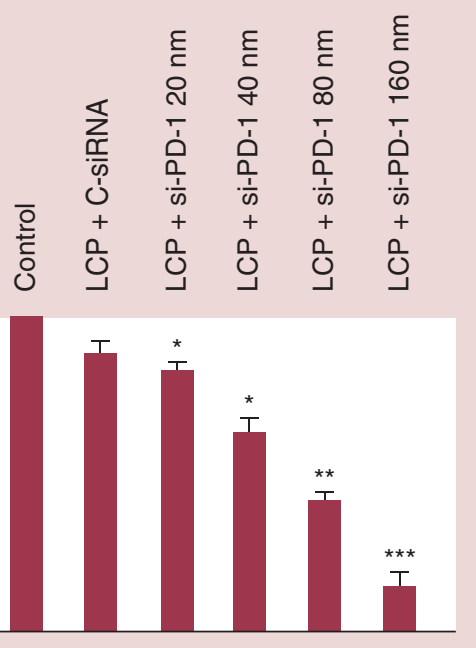

(B)

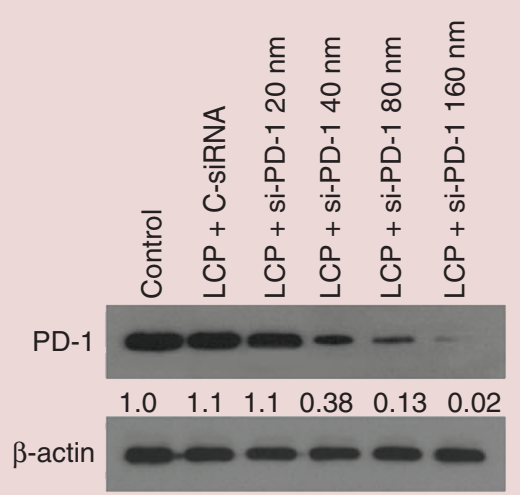

(C)

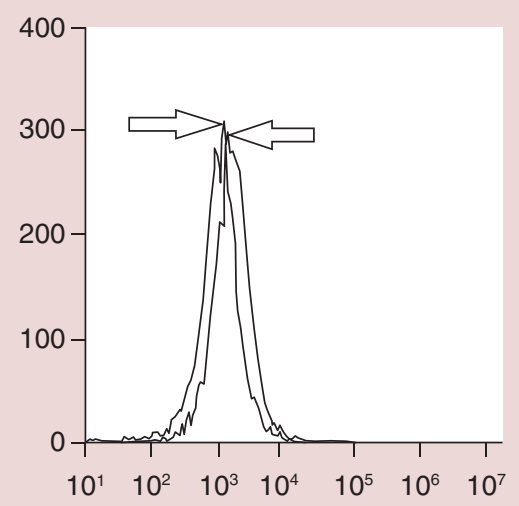

Figure 3. Knocking down PD-1 expression in tumor-infiltrating lymphocytes. (A) RT-PCR data for the knockdown efficiency of PD-1 mRNA in TILs treated with LCP-si-PD-1 at the siRNA concentrations from 20 to $160 \mathrm{nM}$; (B) western blotting assay shows the reduction of PD-1 protein expression in tumor-infiltrating lymphocytes treated with LCP-si-PD-1; (C) flow cytometry analysis of TILs before and after treatment with LCP-si-PD-1 at $40 \mathrm{nM}$. All tests were conducted in three biological repeats. ${ }^{*} \mathrm{p}<0.05 ;{ }^{* *} \mathrm{p}<0.01 ;{ }^{* * *} \mathrm{p}<0.001$. LCP: Lipid-coated calcium phosphate.

of siRNA. The efficient silencing of PD-1 gene can be largely attributed to the high delivery efficacy of LCP NPs, which is consistent with that reported previously [28].

TILs treated with LCP-siRNA-PD-1 at $80 \mathrm{nM}$ of siRNA also showed a significant reduction of PD-1 expression by flow cytometry analysis. As shown in Figure $3 \mathrm{C}$, the fluorescent peak shifted to the lower intensity side, with the mean florescent intensity reduced from 2480 to 960 , in consistence with the reduction of the PD-1 protein 
(A)

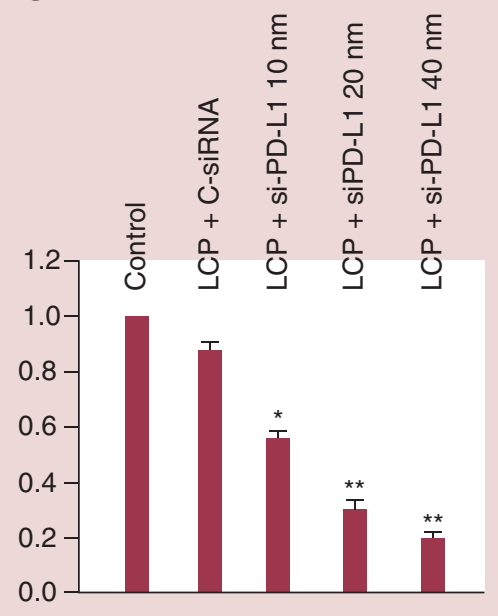

(B)

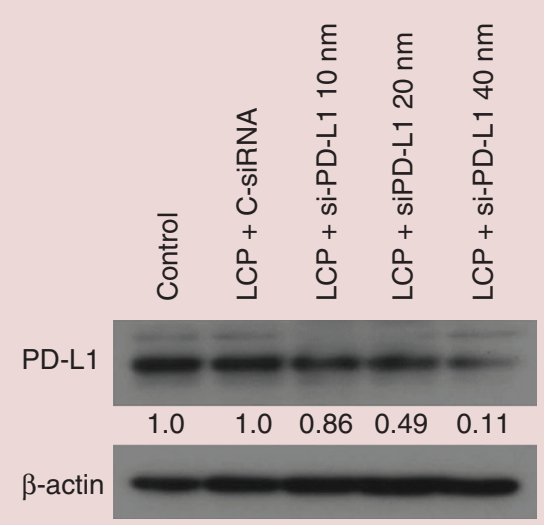

(C)

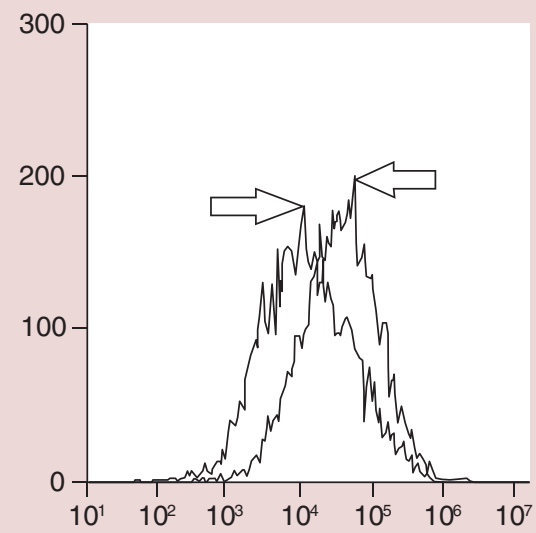

Figure 4. Knocking down PD-L1 in MCF-7 cells. (A) RT-PCR data for the knockdown of PD-L1 mRNA in MCF-7 cells treated with LCP-si-PD-L1 at the siRNA concentrations from 10 to $40 \mathrm{nM}$. (B) Western blotting assay showing the reduction of PD-L1 protein expression in MCF-7 cells treated with LCP-si-PD-L1. (C) Flow cytometry analysis of MCF-7 cells before and after treatment with LCP-si-PD-L1 at 40 nM. All tests were conducted in three biological repeats. ${ }^{*} p<0.05 ;{ }^{*} p<0.01$.

LCP: Lipid-coated calcium phosphate; RT: Real time.

Figure 5. PD-1 or/and PD-L1 knockdown improves the antitumor efficacy of tumor-infiltrating lymphocytes ex vivo. The killing efficacy of tumor-infiltrating lymphocytes (TILs) to MCF-7 cells was measured with cell-mediated cytotoxicity assays, at the TILs/MCF-7 cellular ratios of 10:1, 30:1 and 100:1. PD-1+/PD-L1+: both cells were untreated; PD-1-/PD-L1+: only TILs were treated; PD-1+/PD-L1-: only MCF-7 cells were treated; PD-1-/PD-L1-: both cells were treated. All tests were conducted in three biological repeats. ${ }^{*} p$-value $<0.05$.

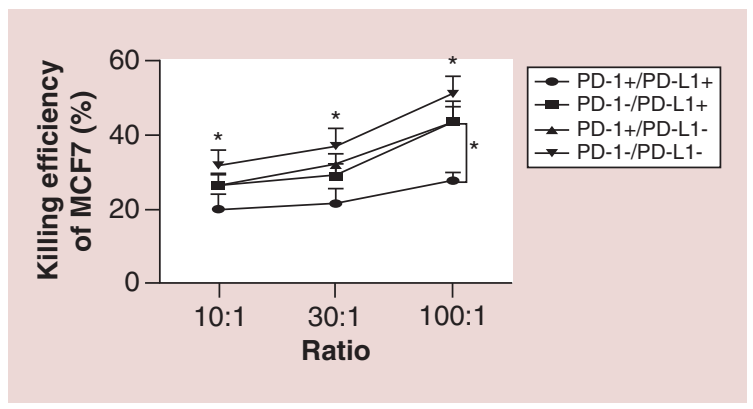

expression (Figure 3B). All the data indicate that siRNA delivered by LCP NPs in this study can effectively downregulate PD-1 expression in TILs.

\section{LCP-si-PD-L1 silencing PD-L1 in MCF-7}

The same delivery system was also used to silence PD-L1 in MCF-7 cells. After treatment with LCP-siPD-L1, the expression levels of $P D-L 1 \mathrm{mRNA}$ and protein in MCF-7 cells were shown in Figure 4. PD-L1 mRNA percentage decreased from 58 to $18 \%$ when the siRNA concentration increased from 10 to $40 \mathrm{nM}$ (Figure 4A). In Figure $4 \mathrm{~B}$, the PD-L1 protein expression level reduced significantly at $40 \mathrm{nM}$ of PD-L1 siRNA. After LCP-si-PD-L1 treatment ( $40 \mathrm{nM}$ ), the fluorescent peak showed a great shift to low fluorescent intensity (Figure 4C). These data demonstrated that siRNA used in this study can effectively knockdown PD-L1 expression in MCF-7. The siRNA doses used for MCF-7 cell transfection were slightly lower than that for TILs (Figure 3A). This was because the transaction efficiency in MCF-7 was about $60 \%$ in terms of positive cell population whereas in TILs it was $50 \%$ (data not shown).

Enhanced killing efficacy after the knockdown of PD-1 or PD-L1 or both

The killing efficacy of TILs to MCF-7 cells after co-culture for $48 \mathrm{~h}$ was shown in Figure 5. The baseline killing efficacy was low $(20 \pm 3 \%)$ when the untreated TILs and MCF-7 cells were used at the TILs/MCF-7 cell number ratio of 10:1. This was slightly increased to $25 \pm 1 \%$ at the ratio of 100:1. For the single downregulation of PD-1 on TILs or PD-L1 on MCF-7, the killing efficacy of TILs was greatly increased from $27 \pm 3 \%$ to $44 \pm 3 \%$ with 


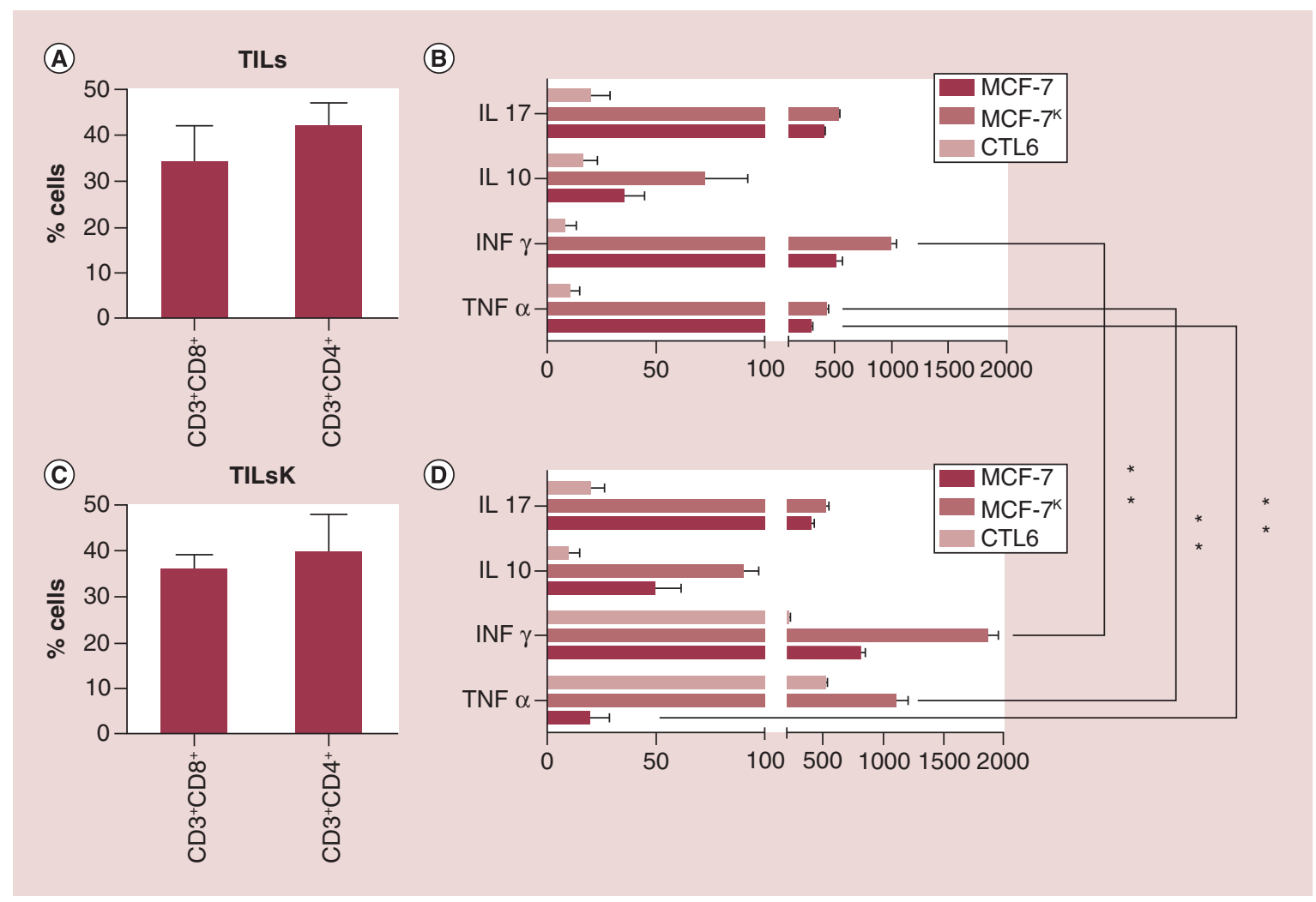

Figure 6. Cytokine production in the co-culture of tumor-infiltrating lymphocytes and MCF-7 cells. Untreated TILs were analyzed for CD4 and CD8 positive cells with $\mathrm{CD} 3$ pan T-cell marker (CD3 ${ }^{+} \mathrm{CD} 4^{+}$or $\mathrm{CD}^{+} \mathrm{CD}^{+}$population) using flow cytometry (A). These cells were co-cultured with MCF-7 cells (MCF-7) for $48 \mathrm{~h}$ and cytokine production was measured in culture supernatants by ELISA (B). Similarly, TILs with PD-1 silenced (TILs ${ }^{\mathrm{K}}$ ) were analyzed for CD4 and CD8 positive cells (C) and were then co-cultured with MCF-7 cells for $48 \mathrm{~h}$. The cytokine production was measured in culture supernatants by ELISA (D). In both assays, the TILs/MCF-7 cell ratio was 100:1. CLT-6, a murine IL-2-dependent cytotoxic T-cell line, was used as a control here. ${ }^{*} p<0.01$.

TIL: Tumor-infiltrating lymphocyte.

the TILs/MCF-7 ratio increasing from 10:1 to 100:1. The efficacy was significantly $(\mathrm{p}<0.05)$ increased at the TILs/MCF-7 ratio of 30:1 and 100:1, compared with that of untreated TILs and MCF-7 cells.

As expected, when both PD-1 and PD-L1 were silenced simultaneously, the killing efficacy improved greatly from $32 \pm 3 \%$ with the ratio of TILs/MCF-7 at 10:1 to $39 \pm 3 \%$ with the TILs/MCF-7 ratio at 30:1 and final to $60 \pm 3 \%$ with the TILs/MCF-7 ratio at 100:1 (Figure 5). These are well above that in all other cases, suggesting a significant $(\mathrm{p}<0.05)$ improvement for the antitumor effect of TILs after knocking down both immune check point molecules (Figure 5).

\section{Measurement of characteristic cytokines after PD-1 or/\& PD-L1 knockdown}

To examine the gene knockdown effect on TIL biofunction and understand the mechanism of above killing ability increase, we measured IFN- $\gamma$, TNF- $\alpha$, IL-17 and IL-10 levels in the co-cultural system. First, we measured CD3 positive CD4 and CD8 phenotypes in TILs (Figure 6A) and their cytokine productions (Figure 6C). We then similarly measured the phenotypes (Figure 6B) and cytokine productions (Figure 6D) of gene silenced TILs (labeled as TIL $^{\mathrm{K}}$ in Figure 6). We did not find any remarkable changes in phenotype (Figure 6A vs C) but find some differences in cytokine production (Figure $6 \mathrm{~B}$ vs D). The differences were that: INF- $\gamma$ increased significantly $(\mathrm{p}<0.01)$ after the gene knockdown of both PD-1 and PD-L1; TNF- $\alpha$ also increased significantly $(\mathrm{p}<0.01)$ after gene knockdown of both PD-1 and PD-L1 but significantly decreased $(\mathrm{p}<0.01)$ after PD-1 knocking down only; IL-17 and IL-10 did not significantly change. 


\section{Discussion}

PD-1-/PD-L1-based cancer immunotherapy is a popular and promising approach for cancer treatment. Using antibodies to block PD-1 and PD-L1 has shown promising outcomes over conventional methods in advanced cancers such as melanoma and lung cancers [34,35]. At present, more and more antibody reagents have been approved by US FDA for clinical application, which will greatly benefit patients suffered with various cancers. In this study, we used a different strategy based on RNAi to silence the gene expression of PD-1 and PD-L1 in T cells and cancer cells. Our data indicate RNAi approach can be an effective way to enhance T-cell cytotoxicity to cancer cells. The use of siRNA to silence PD-1 gene in TILs and enhance their kill efficiency to breast cancer cells and combined knockdown of PD-1 and PD-L1 are new findings that have not yet been reported previously to our best knowledge. Because of the specificity and effectiveness of RNAi-based therapy our study represents a new strategy for PD-1-/PD-L1-based immunotherapy for breast cancer.

The effective delivery of RNAi therapy is a big challenge. In this study, we chose the LCP NPs, they have excellent properties such as high biocompatibility, good biodegradability and low toxicity; [33] thus can be used as an effective and safe siRNA carrier for PD-1/PD-L1 delivery. Another important property of LCP NPs is their excellent ability of endosome escape and quick release of siRNA [36], which ensures effective gene silencing achieved in our study. Indeed, we found that siRNA-mediated downregulation of PD-1 on TILs and PD-L1 on MCF-7 cells significantly enhances the cytotoxicity of TILs to cancer cells. These data suggest that endosome escape has happened in both cell types. Moreover, our strategy will be potentially an alternative or better approach for PD-1-/PD-L1-targeted cancer immunotherapy compared with antibody blocking. For examples, unlike antibodies against cell surface molecules, which have a short half-life and need multiple treatments, RNAi gene silencing can result in a lasting reduction of overall protein expression and consequently lead to a long-term enhancement of killing efficacy of $T$ cells to cancer cells with only one treatment, especially when the lentiviral-shRNA strategy is used in the treatment for long-lasting gene silencing as we reported previously [37].

RNAi therapy is well-known for its effectiveness and low side effects. Therefore, it can be considered as an alternative or a combinational therapy with antibody approach. Although in vivo delivery of siRNA has some issues, such as off-targeted delivery and potential side effects, ex vivo treatment of $\mathrm{T}$ cells especially TILs with siRNA-loaded LCP NPs is an effective and less toxic way to downregulate PD-1 before injecting them back to patients. Our current study has proven that RNAi approach works well ex vivo using LCP NP as the delivery carrier. This strategy combined with PD-L1 antibody treatment will expect a more positive outcome for breast cancer patients. A recent study showed that high levels of PD-1 and PD-L1 expressions are more associated with triple negative breast cancer patients [38], suggesting this strategy will be particularly useful for these patients. For in vivo delivery of siRNA against PD-L1, we can further modify LCP NPs by loading antibody fragment to over-expressed molecules such as HER2 on HER2-positive breast cancer cells, which can further increase the specificity in the future application in clinical setting, as it was reported that PD-L1 expression was positively associated with worse clinical outcome in HER2 positive breast cancer patients [39].

When PD-L1 siRNA delivered by LCP NPs is applied to in vivo settings, the siRNA will not only target cancer cells and silence PD-L1 in these cells, but also possibly target tumor-associated macrophages (TAMs). It has been shown that TAMs express PD-L1 and inhibit T-cell function [40]. Therefore, the possible knockdown of PD-L1 on TAMs will be an extra benefit for PD-L1-based cancer immunotherapy. This is an interesting direction and warrants further investigations. However, the in vivo application of LCP NPs may cause side effects, we thus shall consider more biocompatible NPs such as our reported layered double hydroxides (LDH) [41] or A-C3 polymeric NPs [20] in the future studies.

To further characterize TIL responses to gene silences of $P D-1$ and $P D-L 1$, we analyzed the cytokine production during the co-culture. The most obvious change was the increase of IFN- $\gamma$ production after the gene knocking down (Figure 6). It has been previously shown that TILs with high PD-1 expression could lose their function (exhaust) including reduction of INF- $\gamma$ production [42]. The increase of INF- $\gamma$ in our study is thus believed to relate to the increased activity of TILs after the knockdown of PD-1 or both PD-1 and PD-L1. The reduction of the exhaust factor during the interaction of TILs and MCF-7 cells thus enhances their killing ability, supported by the killing efficiency results (Figure 5).

TNF- $\alpha$ is also a pro-inflammatory cytokine that can induce cell apoptosis, and the increase of its production after PD-1 and PD-L1 knockdown further confirms the enhancement of the TIL function after reducing the inhibitory factors (PD-1 and PD-L1). However, its significant decrease in the co-culture of TIL ${ }^{\mathrm{K}}$ with MCF-7 cells 
is an interesting point, which could be due to the abundant presence of inhibitory PD-L1 by MCF-7. In sharp contrast, silencing PD-L1 on MCF-7 led to a very significant increase of TNF- $\alpha$ production (Figure 6D). This is different from IFN- $\gamma$ response, suggesting that their reaction to PD-L1 inhibition is different, which warrants further investigations.

IL-17 is a cytokine believed to play a promoting role in breast cancer development via increasing chemotherapy resistance and cell proliferation [43]. The presence of Th-17 cells (producing IL-17) [43] or IL-17 [44] is correlated to the poor prognosis in breast cancer patients. IL-10 is another immune-regulatory cytokine, and mainly plays an inhibitory role in $\mathrm{T}$ cell function in tumor microenvironment [45]. We did not observe much changes on these two cytokines before and after gene silence, suggesting that there is no much effect on TILs in these aspects, which is consistent with the increased TIL killing ability.

\section{Conclusion}

Our present study has shown that the combined knockdown of PD-1 and PD-L1 is more effective way than either alone in promoting the killing ability of TILs to cancer cells. Meanwhile, the combined silence of PD-1 and PD-L1 results in increased secretion of pro-inflammatory cytokines such as IFN- $\gamma$ and TNF- $\alpha$. Our research also confirms that LCP NPs are an effective delivery system to T cells and tumor cells. Together these data suggest that simultaneous silence of PD-1 and PD-L1 genes restore TIL effector functions and may also shift the tumor microenvironment from suppressive to pro-inflammatory.

\section{Future perspective}

The Nobel Prize in Physiology or Medicine for 2018 was awarded to 'cancer immunotherapy by inhibition of negative immune regulation'. The research and clinical application of this field are expected to dramatically boom in the next 5-10 years. As the major target of this therapy, TILs-based engineering approach is believed to significantly advance in the due course because of their specificity to cancer cells. Ex vivo expansion of TILs is a powerful way to enhance patient immunity against cancer as personalized medicine. However, TILs' ability is hindered by expression of negative regulators such as PD-1, and ex vivo blockade of these regulators will be an exciting approach to overcome this issue in next few years. Our method reported in the study for gene silencing is certainly an excellent option to achieve this. In our study we prove that engineered TILs show a better ability to kill MCF-7 cells though the finding needs to be expanded to more breast cancer cell lines (e.g., MDA-MB-231 cells) and even orthotopic tumor model to further confirm the results before clinical application.

On the other hand, for cancer cells, PD-L1 gene silence will be a better solution to fully stop PD-L1 from both extracellular and intracellular sources than antibody blockade of only surface PD-L1. Therefore, our reported strategy will be an effective and specific approach for PD-L1-based immunotherapy. However, the issue for this approach is the cancer cell-targeted delivery, especially in vivo settings. In this study, we have shown that NP is a very good choice but still needs to achieve targeted delivery. As many studies from us and others indicate that cancer cell-targeted delivery can be achieved with nanotechnology. Therefore, we believe that targeted PD-L1 gene silencing in cancer cells will be achieved in the next few years, which will lead to the PD-1-/PD-L1-based cancer immunotherapy to be more effective with much lighter side effects. In addition, gene silencing approach will maximize the response rate of cancer patients with a lasting outcome if short-hair RNA is used. In our study, we used LCP NPs that have not been used in clinic due to the cytotoxicity. For future preclinical or clinical studies, more biocompatible NPs will be considered and used. As tumor microenvironment is now known as a protective factor for cancer cells, combining the properties of NPs that can penetrate tumor microenvironment will be also the future consideration.

\section{Financial \& competing interests disclosure}

The authors have no relevant affiliations or financial involvement with any organization or entity with a financial interest in or financial conflict with the subject matter or materials discussed in the manuscript. This includes employment, consultancies, honoraria, stock ownership or options, expert testimony, grants or patents received or pending, or royalties.

No writing assistance was utilized in the production of this manuscript.

Ethical conduct

All the patient-related work was approved by the Research Ethics Committee of the Sun-Yat-Sen University Cancer Center. The authors state that they have obtained appropriate institutional review board approval or have followed the principles outlined in 
the Declaration of Helsinki for all human or animal experimental investigations. In addition, for investigations involving human subjects, informed consent has been obtained from the participants involved.

Informed consent disclosure

The authors state that all patients provided written consent forms before being subjected to blood and/or tumor collection.

\section{Open access}

This work is licensed under the Attribution-NonCommercial-NoDerivatives 4.0 Unported License. To view a copy of this license, visit http://creativecommons.org/licenses/by-nc-nd/4.0/

\section{Availability of data \& material}

All the data and material are available when required.

\section{Summary points}

- Lipid-modified calcium phosphate (LCP) nanoparticles (NPs) were synthesized, and they were round-shaped with positively charged surface.

- LCP NPs have a good capacity to load oligo DNA and siRNAs against human PD-1 and PD-L1 genes.

- Tumor-infiltrating lymphocytes (TILs) were successfully isolated from 15 breast cancer patients and expanded in vitro.

- LCP NPs can efficiently deliver siRNA to TILs and downregulate their PD-1 expression at both mRNA and protein levels.

- LCP NPs can efficiently deliver siRNA to breast cancer MCF-7 cells and downregulate PD-L1 expression at both mRNA and protein levels.

- Either knockdown of PD-1 or PD-L1 can increase the killing efficiency of TILs to breast cancer cells.

- Simultaneous knockdown of PD-1 and PD-L1 can more significantly increase the killing efficiency of TILs to breast cancer cells.

- Knockdown of PD-1 or PD-L1 or both has significant effects on TIL cytokine production profile.

\section{References}

Papers of special note have been highlighted as: $\bullet$ of interest; $\bullet \bullet$ of considerable interest

1. Rosenberg SA, Restifo N. Adoptive cell transfer as personalized immunotherapy for human cancer. Science 348, 62-68 (2015).

2. Nicholas PR, Mark ED, Steven AR. Adoptive immunotherapy for cancer: harnessing the T cell response. Nat. Rev. Immunol. $12(4), 269$ (2012).

3. Steven AR. Cell transfer immunotherapy for metastatic solid cancer - what clinicians need to know. Nat. Rev. Clin. Oncol. 8(10), 577 (2011).

4. Curran KJ, Brentjens RJ. Chimeric antigen receptor T cells for cancer immunotherapy. J. Clin. Oncol. 33(15), 1703 (2015).

5. Curran MA, Kim M, Montalvo W, Al-Shamkhani A, Allison JP. Combination CTLA-4 blockade and 4-1BB activation enhances tumor rejection by increasing T-cell infiltration, proliferation, and cytokine production. PLoS ONE 6(4), e19499 (2011).

6. Gros A, Robbins PF, Yao X et al. PD-1 identifies the patient-specific $\mathrm{CD} 8\left(^{+}\right)$tumor-reactive repertoire infiltrating human tumors. $J$. Clin. Inves. 124(5), 2246-2259 (2014).

- A good example of understanding tumor-infiltrating lymphocytes in melonoma and its relation to PD-1 expression. The in vitro expanded cell population is mostly $\mathrm{CD8}^{+} \mathrm{PD}-\mathrm{L} 1$ tumor-infiltrating lymphocytes.

7. Woo S-R, Turnis ME, Goldberg MV et al. Immune inhibitory molecules LAG-3 and PD-1 synergistically regulate T-cell function to promote tumoral immune escape. Cancer Res. 72(4), 917-927 (2012).

8. Zimmerman N, Gavrieli M, Fallarino F et al. BTLA is a lymphocyte inhibitory receptor with similarities to CTLA-4 and PD-1. Nat. Immunol. 4(7), 670-679 (2003).

9. Kalos M, June CH. Adoptive T cell transfer for cancer immunotherapy in the era of synthetic biology. Immunity 39(1), 49 (2013).

10. Topalian SL, Hodi FS, Brahmer JR et al. Safety, activity, and immune correlates of anti-PD-1 antibody in cancer. N. Engl. J. Med. 366(26), 2443-2454 (2012).

-• A comprehensive clinical study to learn about PD-1 antibody blockade therapy in different cancers.

11. Brahmer JR, Drake CG, Wollner I et al. Phase I study of single-agent anti-programmed death-1 (MDX-1106) in refractory solid tumors: safety, clinical activity, pharmacodynamics, and immunologic correlates. J. Clin. Oncol. 28(19), 3167-3175 (2010). 
12. Abiko K, Mandai M, Hamanishi J et al. PD-L1 on tumor cells is induced in ascites and promotes peritoneal dissemination of ovarian cancer through CTL dysfunction. Clin. Cancer Res. 19(6), 1363-1374 (2013).

- A typical study to show PD-L1 function in inhibiting cytotoxic T-lymphocyte function of peritoneal dissemination of ovarian cancer.

13. Maier H, Isogawa M, Freeman GJ, Chisari FV. PD-1:PD-L1 interactions contribute to the functional suppression of virus-specific CD8+ T lymphocytes in the liver. J. Immunol. 178(5), 2714-2720 (2007).

14. Muenst S, Schaerli AR, Gao F et al. Expression of programmed death ligand 1 (PD-L1) is associated with poor prognosis in human breast cancer. Breast Cancer Res. Treat. 146(1), 15-24 (2014).

15. Schalper KA. PD-L1 expression and tumor-infiltrating lymphocytes: revisiting the antitumor immune response potential in breast cancer. OncoImmunology 3(6), e29288 (2014).

16. Vincenti F. Costimulation blockade in autoimmunity and transplantation. J. Allergy Clin. Immunol. 121(2), 299-306 (2008).

17. Moslehi JJ, Salem J-E, Sosman JA, Lebrun-Vignes B, Johnson DB. Increased reporting of fatal immune checkpoint inhibitor-associated myocarditis. Lancet 391(10124), 933 (2018).

18. Bitko V, Musiyenko A, Shulyayeva O, Barik S. Inhibition of respiratory viruses by nasally administered siRNA. Nat. Med.11, 50-55 (2005).

- This is the first and typical study of RNAi therapy to treat viral infection. It proves that RNAi therapy is safe and effective.

19. Gu W, Cochrane M, Leggatt GR et al. Both treated and untreated tumors are eliminated by short hairpin RNA-based induction of target-specific immune responses. Proc. Natl Acad. Sci. USA 106(20), 8314-8319 (2009).

20. Truong NP, Gu W, Prasadam I et al. An influenza virus-inspired polymer system for the timed release of siRNA. Nat. Communicat. 4 , 1902 (2013).

-• A very good example of siRNA delivery with nanoparticles to efficiently kill cancer cells and silence other genes when the endosome escape mechanism was induced in the delivery system.

21. Scherer LJ, Rossi JJ. Approaches for the sequence-specific knockdown of mRNA. Nat. Biotechnol. 21(12), 1457-1465 (2003).

22. Yuan Z, Nicole Mj S, Arlin BR, William YK, Leaf H. Codelivery of VEGF siRNA and gemcitabine monophosphate in a single nanoparticle formulation for effective treatment of NSCLC. Mol. Ther. 21(8), 1559 (2013).

23. Zhang Y, Kim WY, Huang L. Systemic delivery of gemcitabine triphosphate via LCP nanoparticles for NSCLC and pancreatic cancer therapy. Biomaterials 34(13), 3447-3458 (2013).

-• A very typical study showing the use of lipid-coated calcium phosphate nanoparticle for drug delivery and treatment of cancer $i n$ vitro and in vivo.

24. Tseng Y-C, Xu Z, Guley K, Yuan H, Huang L. Lipid-calcium phosphate nanoparticles for delivery to the lymphatic system and SPECT/CT imaging of lymph node metastases. Biomaterials 35(16), 4688-4698 (2014).

25. Tseng Y-C, Yang A, Huang L. How does the cell overcome LCP nanoparticle-induced calcium toxicity? Mol. Pharm. 10(11), 4391 (2013).

26. Li J, Yang Y, Huang L. Calcium phosphate nanoparticles with an asymmetric lipid bilayer coating for siRNA delivery to the tumor. $J$. Control. Rel. 158(1), 108-114 (2012).

27. Chen Y, Zhu X, Zhang X, Liu B, Huang L. Nanoparticles modified with tumor-targeting scFv deliver siRNA and miRNA for cancer therapy. Mol. Ther. 18(9), 1650-1656 (2010).

28. Tang J, Li L, Howard CB, Mahler SM, Huang L, Xu ZP. Preparation of optimized lipid-coated calcium phosphate nanoparticles for enhanced in vitro gene delivery to breast cancer cells. J. Mater. Chem. B 3(33), 6805-6812 (2015).

29. Jin J, Sabatino M, Somerville R et al. Simplified method of the growth of human tumor infiltrating lymphocytes in gas-permeable flasks to numbers needed for patient treatment. J. Immunother. 35(3), 283-292 (2012).

30. Li J, Chen Q-Y, He J et al. Phase I trial of adoptively transferred tumor-infiltrating lymphocyte immunotherapy following concurrent chemoradiotherapy in patients with locoregionally advanced nasopharyngeal carcinoma. OncoImmunology 4(2), 1-11 (2015).

31. Wu Y, Chen M, Wu P, Chen C, Xu ZP, Gu W. Increased PD-L1 expression in breast and colon cancer stem cells. Clin. Exp. Pharmacol. Physiol. 44(5), 602-604 (2017).

- A good showcase indicating that cancer stem cells expressed high level of PD-L1 that could be a potential target for PD-L1 therapy.

32. Vasievich E, Chen W, Huang L. Enantiospecific adjuvant activity of cationic lipid DOTAP in cancer vaccine. Cancer Immunol. Immunother. 60(5), 629-638 (2011).

33. Tang J, Li L, Howard CB, Mahler SM, Huang L, Xu ZP. Preparation of optimized lipid-coated calcium phosphate nanoparticles for enhanced in vitro gene delivery to breast cancer cells. J. Mater. Chem. B 3(33), 6805-6812 (2015).

34. Luisa C, Sara P, Michele M et al. Differential activity of nivolumab, pembrolizumab and MPDL3280A according to the tumor expression of programmed death-ligand-1 (PD-L1): sensitivity analysis of trials in melanoma, lung and genitourinary cancers. PLoS ONE 10(6), e0130142 
35. Prat A, Navarro A, Paré L et al. Immune-related gene expression profiling after PD-1 blockade in non-small cell lung carcinoma, head and neck squamous cell carcinoma, and melanoma. Cancer Res. 77(13), 3540 (2017).

36. Ma D. Enhancing endosomal escape for nanoparticle mediated siRNA delivery. Nanoscale 6(12), 6415-6425 (2014).

37. Gu W, Putral L, Hengst $\mathrm{K}$ et al. Inhibition of cervical cancer cell growth in vitro and in vivo with lentiviral-vector delivered short hairpin RNA targeting human papillomavirus E6 and E7 oncogenes. Cancer Gene Ther. 13(11), 1023-1032 (2006).

38. Kitano A, Ono M, Yoshida $\mathrm{M}$ et al. Tumour-infiltrating lymphocytes are correlated with higher expression levels of PD-1 and PD-L1 in early breast cancer. ESMO Open 2(2), e000150 (2017).

39. Okabe M, Toh U, Iwakuma $\mathrm{N}$ et al. Predictive factors of the tumor immunological microenvironment for long-term follow-up in early stage breast cancer. Cancer Sci. 108(1), 81-90 (2017).

40. Prima V, Kaliberova LN, Kaliberov S, Curiel DT, Kusmartsev S. COX2/mPGES1/PGE2 pathway regulates PD-L1 expression in tumor-associated macrophages and myeloid-derived suppressor cells. Proc. Natl Acad. Sci. USA 114(5), 1117-1122 (2017).

- Tumor-associated macrophages are positive for PD-L1, which is not only related to cytotoxic T-lymphocyte function inhibition but also as a potential target for PD-L1-based therapy.

41. Li L, Gu W, Chen J, Chen W, Xu ZP. Co-delivery of siRNAs and anti-cancer drugs using layered double hydroxide nanoparticles. Biomaterials 35(10), 3331-3339 (2014).

42. Sakuishi K, Apetoh L, Sullivan JM, Blazar BR, Kuchroo VK, Anderson AC. Targeting Tim-3 and PD-1 pathways to reverse T cell exhaustion and restore anti-tumor immunity. J. Exp. Med. 207(10), 2187-2194 (2010).

43. Cochaud S, Giustiniani J, Thomas C et al. IL-17A is produced by breast cancer TILs and promotes chemoresistance and proliferation through ERK1/2. Sci. Rep. 3, 3456 (2013).

44. Kaewkangsadan V, Verma C, Eremin JM, Cowley G, Ilyas M, Eremin O. Crucial contributions by T lymphocytes (effector, regulatory, and checkpoint inhibitor) and cytokines (TH1, TH2, and TH17) to a pathological complete response induced by neoadjuvant chemotherapy in women with breast cancer. J. Immunol. Res. 2016, 4757405 (2016).

45. Solinas G, Germano G, Mantovani A, Allavena P. Tumor-associated macrophages (TAM) as major players of the cancer-related inflammation. J. Leukoc. Biol. 86(5), 1065-1073 (2009). 
